Annuaire suisse de politique de développement

24-1 | 2005

Faits et statistiques 2005

\title{
5. Politique économique extérieure
}

\section{Gérard Perroulaz}

\section{OpenEdition}

\section{Journals}

Édition électronique

URL : http://journals.openedition.org/aspd/424

DOI : 10.4000/aspd.424

ISSN : 1663-9669

\section{Éditeur}

Institut de hautes études internationales et du développement

\section{Édition imprimée}

Date de publication : 1 avril 2005

Pagination : 55-72

ISSN : 1660-5934

\section{Référence électronique}

Gérard Perroulaz, «5. Politique économique extérieure », Annuaire suisse de politique de développement [En ligne], 24-1 | 2005, mis en ligne le 01 mars 2010, consulté le 07 septembre 2020. URL : http:// journals.openedition.org/aspd/424; DOI : https://doi.org/10.4000/aspd.424 


\section{Politique économique extérieure*}

L A SAUVEGARDE des intérêts de l'économie suisse à l'étranger est l'un des cinq objectifs déclarés de la politique extérieure ${ }^{1}$. La politique économique extérieure comprend les mesures prises par la Confédération pour favoriser les intérêts des exportateurs et investisseurs suisses dans le monde. C'est surtout le Secrétariat d'Etat à l'économie (seco) qui s'occupe des instruments relatifs à la politique économique extérieure, dont un encadré ci-dessous rappelle les principaux instruments. Certains instruments sont analysés dans d'autres chapitres de cet Annuaire (relations financières internationales; commerce international et négociations à l'OMC; place financière suisse et accords bilatéraux avec l'UE sur la fiscalité de l'épargne). Nous nous concentrons dans ce chapitre sur les instruments bilatéraux et sur certains instruments autonomes qui concernent plus particulièrement les relations de la Suisse avec les pays en développement.

Sont analysées dans ce chapitre les propositions du Conseil fédéral et les activités du seco dans le domaine des affaires économiques extérieures, avec une attention particulière sur les accords bilatéraux, le contrôle des exportations et la réforme de la Garantie contre les risques à l'exportation (GRE). La GRE connaît des exercices financiers bénéficiaires depuis de nombreuses années, ce qui a permis de rembourser entièrement l'avance de la Confédération en 2004. La révision de la loi sur la GRE prévoit d'assurer désormais le risque de l'acheteur privé, et non plus uniquement d'achats par des collectivités publiques ou certains clients privés ou semi-privés uniquement lorsqu'ils bénéficient d'une garantie de l'Etat. La GRE actuelle sera transformée en établissement de droit public autonome, qui portera le nom d'Assurance suisse contre les risques à l'exportation (ASRE).

\subsection{Grandes orientations de la politique économique extérieure}

La politique économique extérieure comprend les mesures de la Confédération pour favoriser les relations économiques avec l'étranger et protéger les intérêts des exportateurs et investisseurs suisses dans le monde. Ce chapitre se concentre sur les instruments qui concernent plus particulièrement les relations de la Suisse avec les pays en développement et les pays en transition.

Le Conseil fédéral présente au début de chaque année son Rapport sur la politique économique extérieure aux Chambres fédérales. Ce rapport fait le point sur l'actualité de l'année pour chacun des instruments et traite des relations bilatérales avec les principaux partenaires économiques de la Suisse. Chaque rapport traite plus en profondeur un thème particulier: les liens entre mondialisation, croissance économique et lutte contre la pauvreté dans le rapport 2001;

* Par Gérard Perroulaz, chargé de recherche à l'iuéd.

1 L'objectif de sauvegarde des intérêts de l'économie suisse à l'étranger est inscrit dans la Constitution fédérale (article 101, al. 1). 
l'importance de l'interdépendance économique et des échanges commerciaux pour la croissance économique de la Suisse dans le rapport 2002; la question de la libre circulation des personnes dans l'Union européenne élargie dans le rapport $2003^{2}$; les liens entre politique intérieure et politique extérieure dans le rapport 2004.

\subsubsection{Rapport sur la politique économique extérieure 2004}

Le rapport 2004 présente une vue d'ensemble de la politique économique suisse en mettant en évidence les liens entre la politique extérieure au sens strict, la politique intérieure et la politique de développement. La nécessité d'avoir une telle vue d'ensemble est la thèse centrale du rapport. On ne peut plus dissocier ces trois domaines car leurs interrelations sont importantes ${ }^{3}$. Ces dernières années, le rapport présentait avant tout un regard a posteriori sur les événements de l'année écoulée; le rapport 2004 est beaucoup plus prospectif, en mettant en avant les stratégies du Conseil fédéral dans les domaines de la politique extérieure et intérieure.

Une politique économique intérieure fondamentalement protectionniste est selon le rapport incompatible avec l'ouverture des marchés préconisée à l'extérieur. Une formule utilisée dans le rapport résume la situation: «La politique économique extérieure commence à l'intérieur. $»^{4} \mathrm{Si}$ la Suisse revendique une ouverture des marchés sur le plan international, cela implique en contrepartie une ouverture du marché intérieur aux importations - ce qui signifie également des réformes économiques internes à la Suisse.

Dans ce rapport, le Conseil fédéral détermine les priorités pour la politique économique extérieure: cinq objectifs généraux sont ainsi fixés par la Confédération et précisés sous la forme de quinze lignes directrices. Celles-ci mettent l'accent surtout sur l'accès aux marchés étrangers pour les entreprises suisses, et moins sur l'amélioration de l'accès au marché suisse pour les exportateurs de pays en développement; elles ne concernent que peu les réformes de libéralisation internes à la Suisse. Les cinq objectifs sont les suivants :

$1^{\circ}$ participer activement au renforcement du système économique international; $2^{\circ}$ améliorer l'accès aux marchés étrangers importants;

$3^{\circ}$ ouvrir les marchés étrangers à toutes les catégories économiques;

$4^{\circ}$ améliorer l'accès aux marchés étrangers pour toutes les entreprises, quelle que soit leur taille;

$5^{\circ}$ mettre en œuvre les accords existants et garantir leur application.

Le rapport réaffirme l'importance pour un pays comme la Suisse des négociations multilatérales à l'OMC, notamment celles sur les services (AGCS) et sur les investissements transnationaux. La Suisse souhaite aussi améliorer l'applica-

2 Conseil fédéral, Rapport sur la politique économique extérieure 2003 et Message concernant des accords économiques internationaux du 14 janvier 2004, 112 p. (FF 2004 257).

3 Sources: Conseil fédéral, Rapport sur la politique économique extérieure 2004 et Message concernant des accords économiques internationaux du 12 janvier 2005, disponible sur le site du seco, <www.seco-admin.ch/publikationen/00391/index.html?lang=fr>. Voir aussi: Département fédéral de l'économie, Informations supplémentaires. «Rapport sur la politique économique extérieure 2004 », communiqué de presse, s.d.; «Aussenwirtschaftspolitik ist auch Binnenwirtschaftspolitik», Neue Zürcher Zeitung, 13. Januar 2005.

4 Conseil fédéral, Rapport sur la politique économique extérieure 2004, op. cit., p. 16. 
tion de la protection de la propriété intellectuelle, notamment dans les pays émergents ou en transition.

Le rapport mentionne en outre une série d'obstacles sur cette voie de libéralisation et de défense des intérêts de la Suisse:

• les oppositions internes aux réformes en profondeur et à la libéralisation du marché intérieur, qui réduisent la marge de manœuvre de la Suisse dans les négociations sur l'accès aux marchés;

- les blocages et les lenteurs des négociations dans le cadre de l'OMC;

$\checkmark$ le poids limité de la Suisse dans les négociations avec ses grands concurrents;

- les priorités contradictoires qui peuvent exister entre les alliés dans les négociations (par exemple à l'AELE).

\section{Principaux instruments de la politique économique extérieure}

Instruments multilatéraux

Participation de la Suisse à des organisations et conférences internationales:

- AELE;

- OCDE;

- OMC;

- organisations des Nations unies: CNUCED, ONUDI, OIT, etc.;

- institutions de Bretton Woods (FMl et Groupe de la Banque mondiale);

- organes de surveillance, tel le Groupe d'action financière sur le blanchiment de capitaux (GAFI/OCDE).

Instruments bilatéraux

- Relations Suisse-Union européenne, dont les accords bilatéraux.

- Diplomatie et diplomatie commerciale, visites de conseillers fédéraux à l'étranger et accueil de chefs d'Etat ou de ministres étrangers en Suisse, délégations économiques mixtes (avec des représentants des deux pays en question).

- Accords bilatéraux de libre-échange.

- Accords de promotion et de protection des investissements.

- Conventions de double imposition.

Instruments autonomes

- Promotion des exportations.

- Garantie contre les risques à l'exportation.

- Garantie contre les risques à l'investissement.

- Promotion de la place économique et du tourisme.

- Instruments de coopération au développement (APD gérée par le seco): aide macroéconomique (aide budgétaire et mesures de désendettement), instruments de promotion des investissements, financements mixtes, promotion du commerce, technologie environnementale (cleaner production centers).

- Préférences douanières: droits de douane préférentiels sur l'importation de certains produits en provenance de pays en développement (dans le cadre du Système généralisé de préférences).

- Promotion des importations provenant des pays en développement et en transition, programme SIPPO.

- Contrôle des exportations (lutte contre la prolifération de biens pouvant servir à la production d'armes de destruction massive).

- Mesures d'embargo, mesures de l'ONU, de I'Union européenne, mesures relatives aux «diamants de la guerre».

Source: site Internet du seco, www.seco-admin.ch, sous «Politique économique extérieure». 
La Suisse accorde une très grande importance à la conclusion d'accords bilatéraux avec ses principaux partenaires commerciaux, mais aussi avec les pays représentant de nouveaux marchés potentiels. La conclusion d'accords bilatéraux reste une politique majeure pour la plupart des pays industrialisés, même si se déroulent en parallèle les négociations multilatérales de l'OMC, qui sont par exemple un axe important de la politique économique extérieure de l'Union européenne et des Etats-Unis.

Trois principaux types d'accords bilatéraux ont été conclus par la Suisse depuis de nombreuses années: les accords de protection des investissement, les conventions évitant la double imposition et les accords de libre-échange.

\subsubsection{Accords concernant la promotion et la protection réciproques des investissements}

Chaque partie contractante s'engage à encourager les investissements de l'autre partie. Chaque Etat doit garantir le libre transfert des capitaux et des revenus des investissements : intérêts, dividendes, apports supplémentaires de capitaux, rapatriement des capitaux. La Suisse a signé des accords bilatéraux de ce type avec plus d'une centaine de pays. Les accords avec les pays suivants sont récemment entrés en vigueur (avec entre parenthèses la date de l'entrée en vigueur de l'accord): Singapour (janvier 2003), Nigeria et Kirghizistan (avril 2003), Libye (mai 2004), Mozambique (juillet 2004). Les accords avec les pays suivants ont été signés, mais ne sont pas encore entrés en vigueur (avec entre parenthèses la date de la signature): Qatar (novembre 2001), Guatemala (septembre 2002), Soudan (octobre 2002), Chili (juin 2003), Bosnie-Herzégovine (septembre 2003), République dominicaine (janvier 2004), Tanzanie (avril 2004), Lesotho (juin 2004), Oman (août 2004) et Algérie (30 novembre 2004) .

\subsubsection{Conventions de double imposition}

Ces accords permettent d'éviter que des revenus ne soient imposés à double, dans les deux pays partenaires. Les conventions règlent l'imposition des personnes physiques et des entreprises. Une fois signée par les deux Etats, la convention doit, en Suisse, être approuvée par le Parlement avant ratification. Chaque accord fait donc l'objet d'un message du Conseil fédéral aux Chambres fédérales. Accords récents: le Conseil fédéral a présenté en septembre 2003 son message demandant au Parlement de ratifier la Convention de double imposition avec Israël $1^{6}$. La Convention de double imposition avec l'Iran a été adoptée par le Parlement en juin et en septembre $2003^{7}$ puis est entrée en vigueur le

5 La liste complète des accords concernant la promotion et la protection des investissements conclus à ce jour par la Suisse, avec les dates de signature et d'entrée en vigueur ainsi que des liens vers les textes des accords, se trouve sur le site Internet du seco, <www.seco-admin.ch>, sous «Politique économique extérieure», puis sous «Investissements».

6 Conseil fédéral, Message concernant une Convention de double imposition avec Israël du 19 septembre 2003 (FF 2003 5903).

7 Conseil fédéral, Message concernant une Convention de double imposition avec l'Iran du 19 février 2003 (FF 2003 2311). 
31 décembre 2003. Des négociations sont en cours avec la Bosnie-Herzégovine et le Pérou.

\subsubsection{Accords de libre-échange entre les Etats de l'AELE et des Etats tiers}

Une vingtaine d'accords de libre-échange au total ont été conclus entre l'AELE ${ }^{8}$ et différents Etats d'Europe centrale et orientale, du bassin méditerranéen et de quelques pays d'outre-mer. Dans une première étape, l'AELE et un pays partenaire signent une «déclaration de coopération». Sur cette base, l'accord de libre-échange est ensuite préparé, négocié et signé par l'AELE. Chaque pays membre de l'AELE, dont la Suisse, doit ensuite ratifier l'accord avec le pays en question. Chaque pays de l'AELE conclut des accords bilatéraux séparés pour le secteur agricole. Pour la Suisse, les accords de libre-échange sont importants et sont complémentaires aux négociations menées sur le plan multilatéral au niveau de l'OMC. Le tableau 13 donne la liste des accords de libre-échange conclus ou en négociation.

Tableau 13: Accords de libre-échange entre l'AELE et les pays tiers (état: novembre 2004)

\begin{tabular}{|c|c|c|}
\hline Groupe de pays/région & Accords en vigueur & $\begin{array}{l}\text { Déclaration de coopération } \\
\text { Négociations en cours } \\
\text { (année du début des discussions) }\end{array}$ \\
\hline Pays d'Europe centrale et orientale & $\begin{array}{l}\text { Bulgarie } \\
\text { Croatie } \\
\text { Macédoine } \\
\text { Roumanie }\end{array}$ & $\begin{array}{l}\text { Serbie-et-Monténégro (2000) } \\
\text { Ukraine (2000) }\end{array}$ \\
\hline Etats/territoires du bassin méditerranéen & $\begin{array}{l}\text { Autorité palestinienne } \\
\text { Israël } \\
\text { Jordanie } \\
\text { Liban (2004) } \\
\text { Maroc } \\
\text { Turquie }\end{array}$ & $\begin{array}{l}\text { Albanie (1992) } \\
\text { Algérie (2002) } \\
\text { Egypte (1995) } \\
\text { Tunisie (2004) }\end{array}$ \\
\hline Pays d'outre-mer & $\begin{array}{l}\text { Chili (2004) } \\
\text { Mexique (2001) } \\
\text { Singapour (2003) }\end{array}$ & $\begin{array}{l}\text { Conseil de coopération du Golfe (2000) } \\
\text { MERCOSUR (2000) } \\
\text { Pays de la SACU (2001) } \\
\text { Canada (1998) } \\
\text { Etats-Unis (2004) } \\
\text { Thaïlande (2004) } \\
\text { Japon (2004) } \\
\text { Corée du Sud (2004) }\end{array}$ \\
\hline
\end{tabular}

Sources: à partir des données disponibles sur le site Internet du seco, <www.seco-admin.ch>.

Sur la vingtaine d'accords existants au début de 2004, huit ont disparu en mai 2004, lors de l'élargissement de l'Union européenne. Pour la Suisse, les relations avec ces huit pays relèvent dorénavant des accords bilatéraux SuisseUnion européenne, et pour les autres pays de l'AELE, les domaines sont maintenant couverts par l'espace économique européen.

Suite à l'initiative de l'Union européenne pour mettre en place d'ici 2010 une zone de libre-échange avec les partenaires commerciaux du pourtour méditerranéen, processus dit de Barcelone, il était important pour la Suisse d'éviter que

8 L'AELE comprend les pays suivants, non membres de l'Union européenne: Norvège, Islande, Liechtenstein et Suisse. 
les exportateurs suisses ne soient défavorisés par rapport aux entreprises concurrentes des pays de l'Union européenne. C'est pourquoi la Suisse est favorable à la conclusion de nouveaux accords avec les pays du pourtour méditerranéen. Depuis 1998, l'AELE a commencé à négocier des accords de libre-échange avec des pays d'outre-mer; trois accords ont été conclus jusqu'à maintenant, avec le Mexique (entrée en vigueur le $1^{\text {er }}$ juillet 2001), Singapour (entrée en vigueur le $1^{\text {er }}$ janvier 2003) et le Chili. L'accord avec ce dernier a été signé le 26 juin 2003, le Parlement suisse a approuvé cet accord en décembre 2003 et l'entrée en vigueur a eu lieu en 2004.

Deux nouveaux accords, avec le Liban et la Tunisie, ont été signés en juin 2004 lors de la réunion ministérielle de l'AELE à Montreux. Des négociations sont en cours avec l'Egypte et les pays de l'Union douanière d'Afrique australe (SACU): l'Afrique du Sud, le Botswana, le Lesotho, la Namibie et le Swaziland. Une déclaration de coopération a été adoptée avec le MERCOSUR (Argentine, Brésil, Paraguay et Uruguay) et les pays du Conseil de coopération du Golfe (Arabie saoudite, Bahreïn, Emirats arabes unis, Koweït, Oman, Qatar).

Les accords les plus récents ont des portées très larges, puisqu'ils ne concernent pas uniquement le commerce des marchandises, mais aussi le commerce des services, l'accès au marché pour les investissements, les marchés publics et les règles de protection de la propriété intellectuelle.

\section{$\square$ Revendications des æuvres d'entraide concernant les accords de libre-échange}

La Déclaration de Berne et la Communauté de travail des œuvres d'entraide ont exprimé en juin 2004 une position critique face aux accords de libre-échange9 ${ }^{9}$. Il est pour ces organisations regrettable que la Suisse (ou les Etats-Unis, ou l'Union européenne) donnent une telle importance à des négociations bilatérales, où le pays en développement partenaire est en moins bonne position de force que dans une instance multilatérale. La Suisse profiterait ainsi de ces accords bilatéraux pour exiger des pays en développement un respect plus strict des règles de protection de la propriété intellectuelle que celui qui est nécessaire pour être conforme aux règles de l'OMC.

Deux points, liés aux accords sur la propriété intellectuelle de l'OMC (Accord sur les ADPIC), sont relevés par les organisations d'entraide: les brevets sur les médicaments et l'accès aux semences restreint pour les paysans.

Les accords de libre-échange récents allongent la durée des brevets sur les produits pharmaceutiques au-delà du délai de vingt ans prévu par l'OMC (cinq ans de plus pour la Macédoine et le Chili par exemple, six ans pour le Liban). Cela retarde l'introduction de médicaments génériques, ce qui est inquiétant dans le cadre de la négociation en cours avec l'Afrique du Sud.

Le deuxième point critiqué par les œuvres d'entraide est le fait que certaines dispositions en matière de propriété intellectuelle contenues dans les accords de libre-échange vont au-delà des obligations prévues par l'OMC dans le domaine agricole. Les règles dites «ADPIC-plus» restreignent l'accès aux semences

9 Déclaration de Berne et Communauté de travail des œuvres d'entraide, Bilatéralisme suisse au lieu de multilatéralisme solidaire. Les accords de libre-échange Nord-Sud empêchent le développement, communiqué de presse, 22 juin 2004, <www.ladb.ch>, sous «Communiqués de presse». 
pour les paysans. Les accords récents contiennent par exemple l'obligation pour le pays de respecter la convention de protection des variétés végétales de l'Union internationale pour la protection des obtentions végétales (UPOV), alors que l'Accord de l'OMC sur les ADPIC donne le choix aux Etats membres de protéger les variétés végétales soit par des brevets, soit par un système sui generis (telle la convention de l'UPOV ou une autre formule de protection tenant compte des particularités nationales ${ }^{10}$ ). En signant la convention de l'UPOV de $1991^{11}$, Les paysans voient leur droit de réutiliser les semences limité: l'échange entre agriculteurs de semences protégées est interdit et la réutilisation de semences n'est autorisée que dans la mesure où les intérêts de l'obtenteur ne sont pas lésés.

Des règles pour assurer aux investisseurs suisses l'accès à un pays donné et l'égalité de traitement avec les investisseurs locaux font aussi partie des accords de libre-échange. Pour les organisations d'entraide, il ne faut pas se limiter à demander des droits pour les investisseurs, mais fixer aussi leurs devoirs (respect des conventions fondamentales de l'OIT, responsabilité sociale et environnementale).

Les organisations d'entraide émettent trois revendications :

$\square$ obtenir un processus de consultation transparent sur les accords de libreéchange et d'investissements ;

$\checkmark$ pas de règles allant au-delà des dispositions prévues par l'OMC;

$\square$ ne pas aborder des thèmes refusés par les pays en développement au sein de l'OMC.

\subsection{Garantie contre les risques à l'exportation (GRE)}

La Garantie contre les risques à l'exportation est un instrument permettant aux exportateurs de se protéger contre les risques de non-paiement lors de livraison de biens à l'étranger, lorsque des difficultés de recouvrement sont à craindre en raison de la situation politiquement et économiquement instable dans le pays de l'acheteur. La GRE fonctionnant comme une sorte d'assurance, l'exportateur doit formuler auprès du Bureau pour la GRE une demande de couverture des risques et payer un émolument lié au risque; cette assurance lui permet d'être dédommagé si le client ne paie pas la facture. Les principaux risques couverts par la GRE sont les suivants:

๖ risques politiques, suite à des événements politiques à l'étranger: guerre, émeutes ou révolution;

$\square$ risques de transfert, soit une insuffisance de devises en raison de mesures gouvernementales de contrôle des changes ou suite au rééchelonnement de la dette extérieure d'un pays;

10 D'autres voies que l'UPOV sont explorées, comme par exemple Seeding Solutions (un projet financé partiellement par la DDC), une étude de la FAO ou encore la «loi modèle» élaborée par l'Organisation de l'unité africaine (OUA).

11 Les nouveaux signataires de la convention de l'UPOV n'ont pas d'autre choix que d'adhérer à la Convention révisée en 1991, alors que les anciens membres de 1'UPOV comme la Suisse peuvent choisir de rester sous les dispositions anciennes de la convention, moins contraignantes. 
- risque de ducroire (risque commercial) en cas d'insolvabilité ou de refus de paiement de l'acheteur, qui peut être l'Etat ou une collectivité publique locale, une entreprise publique ou une entreprise privée exécutant une tâche d'utilité publique, telle une centrale électrique ou une usine de traitement des déchets. La GRE ne couvre pour l'instant les risques vis-à-vis d'un acheteur privé que dans certaines conditions (voir ci-dessous «Révision de la loi sur la GRE»);

๖ risques liés à la livraison, si la livraison est rendue impossible en raison de l'augmentation du risque.

\subsubsection{La GRE en chiffres: nouvelles garanties accordées en 2003}

Tableau 14: Répartition géographique des nouvelles garanties et du total des engagements de la GRE en 2003 (en millions de francs et en pourcentage)

\begin{tabular}{|c|c|c|c|c|}
\hline \multirow[t]{2}{*}{ Groupe de pays } & \multicolumn{2}{|c|}{$\begin{array}{c}\text { Nouvelles garanties } \\
\text { en } 2003\end{array}$} & \multicolumn{2}{|c|}{$\begin{array}{c}\text { Total des engagements } \\
\text { au } 31 / 12 / 2003\end{array}$} \\
\hline & Mio. fr. & Part en $\%$ & Mio. fr. & Part en $\%$ \\
\hline \multicolumn{5}{|l|}{ Par continent } \\
\hline Europe & 611.0 & 27.8 & 1903,2 & 21.2 \\
\hline Afrique & 160.0 & 7.3 & 474.2 & 5.3 \\
\hline Asie & 1289,7 & 58.6 & 5393,9 & 60.2 \\
\hline Amérique centrale et du Sud & 140.1 & 6.4 & 1194,0 & 13.3 \\
\hline Total & 2200,8 & 100.0 & 8965,3 & 100.0 \\
\hline \multicolumn{5}{|l|}{ Par catégorie de pays } \\
\hline Pays de I’OCDE & 521.3 & 23.7 & 1620,8 & 18.1 \\
\hline Pays à faible revenu & 68.7 & 3.1 & 933.0 & 10.4 \\
\hline Pays les plus pauvres & 17.2 & 0.8 & 49.6 & 0.6 \\
\hline Autres pays & 1593,6 & 72.4 & 6361,9 & 71.0 \\
\hline
\end{tabular}

Source: GRE, Rapport annuel 2003, 2004, p. 35.

Les nouvelles garanties accordées en 2003 s'élevaient à 2201 millions de francs (1952 millions en 2002). Elles concernaient principalement (par ordre décroissant) la Turquie (525 millions de nouvelles garanties en 2003), Bahreïn (367 millions), l'Iran (334,2 millions), la Chine (183 millions), Israël, l'Arabie saoudite, l'Algérie et le Mexique. Comme ces dernières années, c'est l'Asie qui représente la plus grande part du total des nouvelles garanties, soit près de $59 \%$, suivie de l'Europe (28\%). Peu de demandes de couverture des exportations concernent le continent africain ou le groupe des pays les plus pauvres, ce qui reflète la faiblesse des relations économiques entre la Suisse et ces groupes de pays pauvres. Le rapport annuel 2003 relève que «les possibilités de [la GRE] d'encourager le développement économique de ces pays sont très limitées. En raison des conditions-cadres qui nous régissent, nous ne sommes souvent pas non plus à même d'assurer le risque d'un engagement dans ces pays, qui restent donc dépendants des fonds de développement ${ }^{12}$. Comme les années précédentes, l'exercice 2003 s'est terminé avec un bénéfice (224 millions de francs). Ce résultat positif permet à la GRE de rembourser progressivement l'avance de la Confédération, et les pertes ont été ainsi totalement épongées en juin 2004. $60 \%$ de toutes les nouvelles garanties accordées sont inférieures à la limite des 5 millions de francs, ce qui montre que la GRE est beaucoup sollicitée par des

12 GRE, Rapport annuel 2003, Berne; Zurich, 2004, p. 11. 
PME. $65 \%$ des nouvelles garanties concernent l'industrie des machines et $34 \%$ la chimie. Dans l'industrie des machines, $62 \%$ des nouvelles garanties concernent le secteur de la fabrication et la distribution d'électricité, et $21 \%$ les machines textiles.

Le graphique 1 montre quels sont les pays les plus concernés par une couverture par la GRE. La Turquie, l'Iran, la Chine et Bahreïn représentent la moitié du total des engagements de la GRE fin 2003. Les engagements totaux de la GRE envers ces quatre pays sont en nette augmentation de 1999 à 2003, ayant passé de 2,6 milliards de francs en 1999 à 4,5 milliards fin 2003. L'Indonésie était, à la fin de la présidence de Suharto, le principal pays bénéficiaire de la GRE avec un engagement de plus d'un milliard de francs au début des années 1990, mais les engagements totaux envers ce pays ont nettement diminué ces dernières années et l'Indonésie s'est retrouvée au sixième rang des pays avec les engagements les plus élevés (369 millions de francs fin 2003).

\section{Graphique 1: Les 15 pays principaux couverts par la GRE:} montant total des engagements au 31 décembre 2003 (en millions de francs)

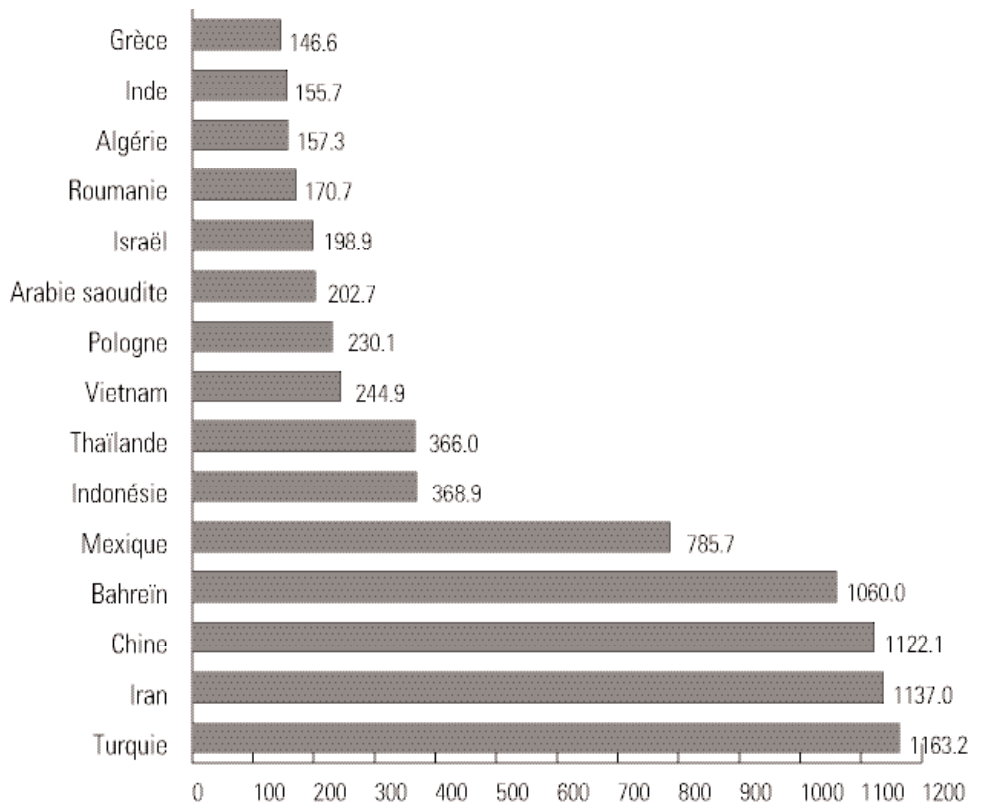

Source: graphique élaboré à partir de: GRE, Rapport annuel 2003, 2004, pp. 41-43.

Sous réserve de l'acceptation de l'entreprise concernée, tous les projets couverts par la GRE dépassant le montant de 10 millions de francs figurent désormais sur le site Internet de la $\mathrm{GRE}^{13}$. Cette liste permet de voir que ce sont quelques grands projets controversés comme les grands barrages qui sont médiatisés. La documentation publiée par le Bureau pour la GRE donne en revanche une longue liste d'exemples de demandes de couverture par des PME, ce qui montre

13 <www.swiss-erg.com>. 
l'importance du soutien aux exportations dans des pays où les risques de nonpaiement sont élevés pour les petites et moyennes entreprises ${ }^{14}$.

\subsubsection{La révision de la loi sur la GRE}

\section{$\square$ Principales réformes du système de garantie}

Le Conseil fédéral a présenté, le 24 septembre 2004, le message et la nouvelle Loi fédérale sur l'Assurance suisse contre les risques à l'exportation (LARE), qui remplacera la loi sur la GRE ${ }^{15}$. Le rapport sur la procédure de consultation sur le projet de réforme, menée en hiver 2003-2004 auprès des milieux intéressés, était paru en juin $2004^{16}$. La révision du système de garantie contre les risques à l'exportation porte essentiellement sur deux points: l'extension de la couverture au risque de l'acheteur privé et la mise en place d'un établissement de droit public. Le projet est examiné par les Chambres fédérales en 2005.

La GRE ne couvre actuellement pas le risque de ducroire privé; un acheteur privé ne peut être couvert par la GRE uniquement s'il bénéficie d'une garantie de paiement d'une banque agréée par la GRE ou d'une garantie de l'Etat. En cas de non-paiement, la Confédération traite directement le cas avec l'Etat en question. Les arguments principaux avancés par le Conseil fédéral pour étendre l'assurance à des acheteurs privés sont les suivants :

- la privatisation de secteurs économiques auparavant dans les mains du secteur public s'est poursuivie dans les pays en développement, les pays émergents et les pays en transition. Les acheteurs privés ne peuvent souvent pas obtenir des garanties de banques locales;

- les institutions comparables de garantie contre les risques à l'exportation d'autres pays industrialisés permettent de couvrir le risque de l'acheteur privé. L'absence d'une offre comparable pour les exportateurs suisses constitue un désavantage pour la place économique suisse;

๖ l'offre d'assurances comparables est inexistante sur le marché privé des assurances.

La GRE était jusqu'à maintenant un fonds de la Confédération sans personnalité juridique et le Bureau pour la GRE était rattaché à la Société suisse des constructeurs de machines (Zurich). Pour la nouvelle assurance, le Conseil fédéral a examiné plusieurs formes juridiques possibles de privatisation ou de partenariat public-privé (PPP). Les enquêtes menées en Suisse ont révélé que la participation à un PPP n'intéressait pas les milieux économiques. Les formes d'institutions sont très variées dans d'autres pays: il peut s'agir d'entreprises publiques indépendantes (comme en Autriche, en Grande-Bretagne ou en Suède), ou encore de confier un mandat de prestations à une entreprise privée

14 Le Rapport annuel 2003 donne l'exemple de deux entreprises pour lesquelles la GRE est très importante: Rieter Textile Systems, pour l'exportation de machines à broder ou de machines textiles en Turquie, et BOBST SA, un des leaders mondiaux dans le domaine de l'équipement et des services pour l'industrie de l'emballage, avec des exportations dans une cinquantaine de pays.

15 Conseil fédéral, Message concernant la Loi fédérale sur l'Assurance suisse contre les risques à l'exportation du 24 septembre 2004 (FF 2004 5441) et Loi fédérale sur l'Assurance suisse contre les risques à l'exportation (FF 2004 5497).

16 seco, Loi fédérale sur l'Assurance suisse contre les risques à l'exportation (LARE). Rapport du DFE sur la procédure de consultation, juin 2004. 
(comme en Allemagne, en France ou aux Pays-Bas). Le Conseil fédéral a choisi la création d'un établissement de droit public pour l'organisation de l'assurance, ce qui permet de laisser au Conseil fédéral et au Parlement la possibilité d'exercer une influence sur son fonctionnement ${ }^{17}$. Le Conseil fédéral pourra formuler les objectifs stratégiques de l'ASRE et déterminer un plafond d'engagement; il prendra les décisions sur les cas d'assurance de portée et d'importance particulières. Il restera aussi compétent pour les accords de rééchelonnement de la dette et pour les accords de réassurance avec d'autres pays. Le Parlement (et le grand public) pourra s'informer au moyen du rapport de gestion et des comptes annuels, documents qui devront être approuvés par la Confédération.

\section{$\square$ Réactions des milieux intéressés lors de la procédure de consultation}

Tous les cantons, les milieux économiques, les syndicats et la majorité des partis politiques se sont exprimés en faveur de l'introduction de la couverture du risque de l'acheteur privé. Seuls le PS, l'UDC, le PCS (Parti chrétien social suisse) et la plupart des ONG se sont exprimés contre l'extension de la couverture à l'acheteur privé. Selon eux, ce risque devrait être couvert par des assurances privées; un argument qu'ils avancent fréquemment est que cette extension introduit une sorte de subvention aux exportateurs et que l'on aboutirait à une situation de privatisation des profits et de socialisation des pertes. Des doutes ont été exprimés sur la possibilité d'autofinancer la couverture de ce risque, la crainte étant que la GRE ne redevienne déficitaire et ne doive demander à nouveau à terme une aide financière de la Confédération. L'examen de la capacité de paiement d'un client étranger sera en effet difficile et les frais administratifs pour l'examen des projets seront plus grands, surtout si l'économie privée recourt massivement à cette nouvelle couverture. La Déclaration de Berne rappelle que la GRE était déficitaire jusqu'à la fin des années 1980 et qu'elle a été aidée grâce à l'intervention de la Confédération :

๖ avance de la Confédération (prêts de 2,5 milliards de francs au maximum en $1992)^{18}$

- abandon d'une partie des créances en 1990 (900 millions supprimés par le Parlement pour éponger les déficits de la couverture des risques monétaires ${ }^{19}$;

口 cession d'une partie des créances envers les pays en développement à la Confédération dans le cadre du programme de désendettement (327 millions de francs).

Assurer le risque de l'acheteur privé contredit, selon la Déclaration de Berne, le principe de subsidiarité prôné dans d'autres domaines par les milieux écono-

17 La forme juridique de l'ASRE, un établissement de droit public, est comparable à celle l'Institut fédéral de la propriété intellectuelle, des écoles polytechniques fédérales ou de la Caisse nationale suisse en cas d'accidents (CNA).

18 L'avance de la Confédération s'élevait encore à presque 2 milliards de francs en 1994. Elle a ensuite régulièrement diminué, notamment par les exercices annuels bénéficiaires (encaissements des primes d'assurance versées par les entreprises d'exportation et des intérêts plus élevés que le versement des dommages lors de cessation de paiement). L'avance a ainsi baissé, aussi en raison de l'abandon d'une partie de la créance et des opérations de désendettement, à 900 millions de francs fin 1998 et à 150 millions de francs fin 2003. Les dettes envers la Confédération ont été épongées en juin 2004.

19 L'introduction de la couverture du risque monétaire dans les années 1970 avait entraîné des pertes de change de 900 millions de francs jusqu'au début des années 1980, lorsque cette couverture a été supprimée. 
miques: l'Etat ne devrait ainsi pas intervenir dans le marché quand l'économie privée pourrait assumer ce risque par des assurances privées.

Pour economiesuisse, une assurance contre les risques à l'exportation reste un instrument indispensable pour une économie tournée largement vers l'extérieur et le projet de loi tient compte des exigences posées par economiesuisse en matière d'orthodoxie libérale, sans charges financières supplémentaires pour la Confédération $^{20}$. Il est important pour la Suisse d'harmoniser la GRE avec les assurances à l'exportation existantes dans les pays concurrents, en couvrant comme les autres le risque de l'acheteur privé.

Le seco estime que le personnel de l'ASRE devra passer des quatorze postes actuels à vingt-huit postes, notamment pour faire face à l'examen des dossiers concernant le risque de l'acheteur privé.

\section{$\square$ Prise en compte des intérêts de politique extérieure}

La décision de l'octroi ou non d'une assurance contre les risque à l'exportation repose surtout sur une analyse financière du projet. La loi impose à l'ASRE «de tenir compte des principes de la politique étrangère de la Suisse». Selon le Conseil fédéral, «du point de vue de la politique de l'Etat, la prise en compte de la politique de développement ne suffit plus de nos jours. Il faut tenir compte de la totalité des domaines relevant de la politique extérieure, notamment les droits de l'homme, la politique de promotion de la paix, la politique environnementale et la politique de développement (coopération au développement et avec l'Europe de l'Est) $»^{21}$.

La Déclaration de Berne salue le fait que l'ASRE doive tenir compte des principes de base de la politique étrangère, mais souhaitait une formulation beaucoup plus contraignante que «tenir compte». La loi devrait ainsi explicitement dire que l'assurance ne doit pas être en contradiction avec ces principes, alors que le Conseil fédéral propose que cela soit fixé dans l'ordonnance d'application.

Lors de la procédure de consultation, les $\mathrm{ONG}^{22}$, l'USS et le PS ont estimé que le projet de loi ne précisait pas suffisamment les principes de la politique étrangère de la Suisse qui doivent être respectés en termes de développement, de politique sociale, de droits de l'homme et de protection de l'environnement. MERS, la plate-forme des organisations actives pour la promotion des droits de l'homme, a relevé que les obligations relatives au respect des droits de l'homme étaient insuffisantes. Les ONG demandaient que le respect des droits de l'homme soit une condition sine qua non de la conclusion d'un contrat d'assurance. Pro Natura et la Déclaration de Berne réclamaient le respect des normes sociales et environnementales. Le PS, Transparency International, Amnesty et la Déclaration de Berne souhaitaient obliger l'exportateur à confirmer par écrit qu'aucun pot-de-vin n'a été versé dans le cadre du contrat de vente. Le projet de loi contient des formules moins explicites et trop vagues pour les ONG. L'article $16 \mathrm{du}$ projet de loi contient l'obligation d'informer et le devoir de

20 economiesuisse, Newsletter, $\mathrm{n}^{\circ} 4$, avril 2004, p. 3.

21 Conseil fédéral, Message concernant la Loi fédérale sur l'Assurance suisse contre les risques à l'exportation du 24 septembre 2004, op. cit., p. 5473.

22 Les ONG suivantes ont émis une prise de position critique face au projet du Conseil fédéral : Amnesty International, la Communauté de travail des œuvres d'entraide, la Déclaration de Berne, Menschenrechte Schweiz MERS, Pro Natura et Transparency International Suisse. 
diligence: «La personne qui veut contracter, ou qui a contracté, une assurance est tenue de fournir et de faire vérifier les renseignements nécessaires à l'estimation de l'opération d'exportation et au suivi du contrat d'assurance.» Les prestations sont exclues si «l'assurance a été conclue sur la base de fausses indications » ou «lorsque le preneur d'assurance enfreint les clauses du contrat d'assurance ou qu'il subit des pertes en raison d'un comportement contraire au contrat conclu avec le débiteur» (article 18). L'exportateur doit ainsi respecter certaines conditions: clause anticorruption, données à fournir relatives aux projets qui sont délicats sur le plan de l'environnement ou du développement ${ }^{23}$.

En ce qui concerne les conditions de travail en Suisse, le PS et les syndicats demandaient qu'un contrat d'assurance ne puisse être conclu que si les conditions de travail usuelles dans la branche étaient respectées. Les organisations demandaient aussi une meilleure transparence ex ante sur les demandes de garanties en cours et sur les études d'effets à long terme sur le développement, la politique étrangère et l'environnement.

Pour l'économie privée, la couverture du risque de l'acheteur privé est importante pour plusieurs raisons: la libéralisation de l'économie et la privatisation se poursuivent dans le monde, le financement à long terme ne peut pas être couvert par le marché des assurances privées, l'économie suisse ne doit pas être défavorisée par rapport aux entreprises d'autres pays du Nord, et l'industrie suisse des machines ainsi que la chimie dépendent largement des exportations.

\section{$\square$ Composition du nouveau Conseil d'administration}

Le Conseil d'administration de l'ASRE habilité à choisir les projets assurés sera composé de sept à neuf membres, choisis pour leurs compétences spécialisées nécessaires à la gestion d'une assurance. Les membres devront, selon le message du Conseil fédéral, avoir aussi des connaissances du domaine des exportations et être en mesure d'évaluer la compatibilité des affaires avec les principes de la politique étrangère.

Le PS, les syndicats et les ONG ont critiqué la composition très technique et économique du futur Conseil d'administration, car elle constitue une régression par rapport à la composition actuelle de la Commission de la GRE. Des représentants de la Confédération, des partenaires sociaux et de la société civile organisée devraient être assurés de pouvoir siéger dans le Conseil d'administration.

L'ancienne loi sur la GRE prévoyait une compétence d'accorder les garanties à différents échelons. Selon l'importance croissante de l'affaire, la proposition de la Commission de la GRE devait être avalisée soit par le seco (projets de moins de 5 millions de francs), soit par le Département fédéral de l'économie seul (projets de 5 à 10 millions), soit par le DFE avec le consentement du Département fédéral des finances (DFF) (projets supérieurs à 10 millions de francs), soit par le Conseil fédéral (pour les projets d'une importance particulière). Dorénavant, l'ASRE aura en principe la compétence de décider de toutes les demandes (article 35). Pour une affaire importante, par exemple un grand barrage aux répercussions sociales et écologiques importantes, le Conseil fédéral pourra donner des instructions à l'ASRE, sur demande du département compé-

23 Conseil fédéral, Message concernant la Loi fédérale sur l'Assurance suisse contre les risques à l'exportation du 24 septembre 2004, op. cit., p. 5483. 
tent (DFE). Il pourrait ainsi obliger l'ASRE à ne pas accorder une couverture lorsque des intérêts supérieurs dominent.

Le fait que le seul département habilité à porter un cas controversé pour arbitrage jusqu'au Conseil fédéral soit le Département de l'économie a été critiqué pendant la procédure de consultation par la Déclaration de Berne, car cela enlève la possibilité au Département fédéral des affaires étrangères (DFAE) et à la Direction du développement et de la coopération (DDC) en particulier d'exprimer suffisamment tôt des interrogations sous l'angle de la cohérence entre les politiques suisses à l'égard des pays en développement. Tous les départements ou offices concernés de l'administration fédérale devraient pouvoir être consultés et examiner les questions de cohérence.

Suite aux critiques émises sur ce point pendant la procédure de consultation, le Conseil fédéral a ajouté à l'article 27 de la loi la possibilité, pour le Conseil fédéral, d'instituer un organe consultatif de représentants de la Confédération, des milieux économiques, des partenaires sociaux et des autres milieux intéressés. Cet organe pourra assister le Conseil d'administration pour les affaires touchant à la politique extérieure ou ayant une portée particulière. L'organisation de cette commission consultative pourra être précisée par voie d'ordonnance et le Conseil fédéral pourra révoquer cet instrument s'il s'avère inutile.

\subsubsection{GRE et questions de cohérence entre la promotion des exportations et le développement durable}

La GRE n'est pas un instrument de coopération au développement, mais vise à encourager les exportations vers des marchés réputés plus risqués et à sauvegarder ainsi des emplois en Suisse. Depuis les années 1970, l'octroi de garanties pour certaines exportations controversées vers les pays en développement a pourtant fait l'objet de critiques régulières de la part de certaines ONG de développement. De nombreuses études relèvent les conflits d'intérêts qui peuvent survenir entre d'une part les objectifs de promotion du développement durable et des droits de l'homme, et d'autre part les objectifs de promotion des exportations ${ }^{24}$.

메 Annuaire 2003, $\mathrm{n}^{\circ}$ 1, pp. 57-59.

Plusieurs projets ont été régulièrement critiqués par les œuvres d'entraide: la fourniture de turbines pour des grand barrages (barrage des Trois Gorges en Chine, barrage d'Illisu en Turquie et, plus récemment, le projet de barrage de Bujagali, en Ouganda) ou pour des centrales électriques, ou encore un équipement de décryptage pour les services secrets en Indonésie. Les ONG revendiquent depuis longtemps une meilleure cohérence avec les objectifs de développement. Même si la GRE n'est pas un instrument de coopération, les ONG ont demandé à de nombreuses reprises de renoncer à des projets contestables sur les

24 Voir par exemple les études suivantes, qui relèvent les incohérences possibles entre le système de Garantie contre les risques à l'exportation et des objectifs de la politique extérieures: J. Forster and O. Stokke (eds.), Policy Coherence in Development Co-operation, EADI Book Series, $\mathrm{n}^{\circ} 22$, London, Frank Cass, 1999, 499 p. L. Goetschel, M. Bernath und D. Schwarz, Schweizerische Aussenpolitik. Grundlagen und Möglichkeiten, Zürich, Verlag NZZ, 2002 (GRE: pp. 207-211); traduction française : Politique extérieure suisse. Fondements et possibilités, Lausanne, Payot, 2004. E. Schläppi und W. Kälin, Schweizerische Aussenwirtschaftshilfe und Menschenrechtspolitik. Konflikte und Konvergenzen, Chur; Zürich: Verlag Rüegger, 2001, 288 p. 
plans de la politique de développement, des droits de l'homme ou du développement durable. Dans la révision de la loi sur la GRE de 1981, le Conseil fédéral avait stipulé que les objectifs de la politique de coopération au développement devaient être respectés dans le cas d'exportations vers les pays les plus pauvres. La Commission de la GRE a été progressivement élargie pour intégrer des représentants de syndicats, puis un représentant de la DDC, mais le Conseil fédéral s'est toujours refusé à accepter un représentant d'ONG de développement.

\subsection{Contrôle des exportations et sanctions économiques}

\subsubsection{Exportations d'armes}

Par les contrôles des exportations de matériel de guerre, la Confédération souhaite veiller au respect des obligations internationales ${ }^{25}$ et des principes de la politique étrangère de la Suisse, tout en cherchant à maintenir en Suisse une capacité industrielle utile aux besoins de la défense nationale. En Suisse, la Loi sur le contrôle des biens à double usage (LCB) et la Loi sur le matériel de guerre (LMG) permettent un contrôle des exportations de ces biens. C'est le seco qui est chargé de l'exécution des contrôles et qui délivre les autorisations d'exporter. La Confédération autorise l'exportation de matériel de guerre si celle-ci ne contrevient pas au droit international et n'est pas contraire aux principes de la politique étrangère suisse.

Les exportations d'armes avaient progressivement diminué de 1987 à 1995, en passant de 575 millions de francs en 1987 à 130 millions de francs en $1995^{26}$. Ces exportations ont à nouveau progressé jusqu'en 2003, pour atteindre 379 millions de francs. Les exportations d'armes sont surtout destinées à des pays industrialisés; l'Allemagne, l'Espagne et la Suède, représentant $45 \%$ des exportations totales de la Suisse, sont ainsi les meilleurs clients des entreprises suisses, mais on trouve aussi près d'une quarantaine de pays en transition ou en développement, dont les principaux étaient en 2003 le Botswana (achats pour un montant de 32,5 millions de francs, au $4^{\mathrm{e}}$ rang des meilleurs clients de la Suisse), les Emirats arabes unis (18 millions), la Malaisie (8,7 millions), Singapour, Bahreïn et l'Arabie saoudite. Des armes ont aussi été exportées en 2003 (pour de modestes montants) à la Roumanie, la Jordanie, les Philippines, l'Egypte, la Bosnie, la Corée du Sud, la Turquie et l'Indonésie. Il faut toutefois relever que les exportations d'armes représentent une très petite part du total des exportations suisses : 0,86\% des exportations totales en 1987, 0,15\% en 1995 et part remontant à $0,28 \%$ en 2003. En 2003,30\% des exportations étaient constituées de véhicules blindés, $21 \%$ de matériel de conduite de tir et $20 \%$ de munitions. La même année, plus de 2000 demandes de permis d'exportation ont été déposées, pour un montant de près d'un milliard de francs, en vertu du contrôle

25 La Suisse doit notamment veiller aux engagements pris par les conventions multilatérales de désarmement qu'elle a ratifiées, avec des contrôles des exportations: Traité sur la non-prolifération des armes nucléaires (TNP), Convention sur les armes chimiques (CSC), Convention sur les armes biologiques (CAB).

26 Sources des chiffres sur les exportations d'armes: site Internet du seco, <www.seco-admin.ch>, sous «Politique économique extérieure», puis sous «Contrôles à l'exportation», puis sous «Matériel de guerre». 
prévu par la Loi sur le matériel de guerre ${ }^{27}$. Seize demandes ont été refusées en 2003, pour un montant de 1,9 million et concernant douze pays. La grande différence entre la valeur réelle de l'exportation et le montant total des demandes acceptées s'explique par le fait que le matériel autorisé une année peut n'être exporté que l'année suivante ou par le fait que certaines commandes peuvent finalement être annulées par le client.

Si les exportations de matériel de guerre sont peu importantes (voir les 379 millions de francs pour 2003 détaillés ci-dessus), la Suisse fait par contre partie de la demi-douzaine de pays qui exportent le plus de biens à double usage, pour un montant annuel de 3 à 4 milliards de francs. La Loi sur le contrôle des biens ${ }^{28}$ permet de contrôler les exportations de biens à double usage, civil ou militaire. Des machines-outils, des simulateurs de vol, des produits chimiques peuvent par exemple être utilisés soit pour un usage civil, soit pour l'armée. La Suisse cherche à empêcher la fabrication d'armes de destruction massive et à veiller au respect des conventions internationales, telles que la Convention sur les armes chimiques. Elle tente aussi d'éviter que l'industrie suisse d'exportation ne soit désavantagée par rapport à des concurrents d'autres pays. Depuis début octobre 2002 jusqu'à fin septembre 2003, le seco a donné des autorisations d'exporter pour un montant total de 514 millions de francs, en vertu de l'Ordonnance sur le contrôle des biens. Deux demandes, relevant l'une du domaine nucléaire, l'autre $\mathrm{du}$ domaine des armes biologiques, ont été refusées, pour un montant de 1,2 million de francs. Deux cas de violation de la LCB ont été dénoncés par le seco auprès du Ministère public de la Confédération pendant cette période ${ }^{29}$.

Dans son rapport sur la politique de maîtrise des armements, paru en septembre 2004, le Conseil fédéral reconnaît que les contrôles à l'exportation n'ont qu'une efficacité limitée sur la maîtrise des armements, notamment pour les raisons suivantes : ${ }^{30}$

๖ les contrôles peuvent entraver l'acquisition d'armes sans toutefois l'empêcher complètement. Les utilisateurs finaux peuvent se procurer les technologies soumises au contrôle par d'autres canaux ;

- l'efficacité des contrôles a tendance à décroître car certains pays concernés peuvent disposer de plus en plus du savoir technologique nécessaire et l'échanger entre eux (prolifération Sud-Sud). Les pays industrialisés n'ont plus le monopole des technologies clés des armes de destruction massive, comme l'attestent par exemple un marché noir international de la technologie nucléaire ou des capacité de développement de technologies nucléaires au Sud (comme en Corée du Nord, en Iran ou en Libye);

๖ les listes de biens à soumettre au contrôle des exportations suivent l'évolution technologique avec retard;

27 Loi fédérale du 13 décembre 1996 sur le matériel de guerre (RS 514.51). Les critères d'autorisation sont détaillés dans l'Ordonnance du 15 février 1998 sur le matériel de guerre (RS 514.511).

28 Loi fédérale du 13 décembre 1996 sur le contrôle des biens utilisables à des fins civiles et militaires et des biens militaires spécifiques (RS 946.202).

29 Conseil fédéral, Rapport sur la politique économique extérieure 2003, op. cit., pp. 353-354.

30 Rapport du Conseil fédéral sur la politique de maîtrise des armements et de désarmement de la Suisse 2004 (en réponse au postulat Haering 02.3541 du 2 octobre 2002) du 8 septembre 2004 (FF 2004 4833), pp. 4848-4849. 
- la Suisse doute de l'efficacité des contrôles des exportations pour empêcher les groupes terroristes d'accéder à certaines armes, car la lutte contre le terrorisme relève plutôt du travail de la police et des renseignements.

[D Annuaire 2005, $\mathrm{n}^{\mathrm{o}}$ 1, dans le chap. 11, point 11.2.1., voir «Politique de maîtrise des armements et de désarmement de la Suisse».

\subsubsection{Sanctions de la Suisse}

En vertu de la Loi sur les embargos, le Conseil fédéral a la compétence pour édicter les mesures de sanction, notamment pour faire respecter le droit international public, issu par exemple de l'ONU ou de l'Organisation pour la sécurité et la coopération en Europe.

En 2004, la liste des sanctions existantes comprenait des mesures contre certains pays (Irak, Yougoslavie, Liberia, Myanmar, Sierra Leone et Zimbabwe) ainsi que les «mesures à l'encontre de personnes et entités liées à Oussama ben Laden, au groupe "Al-Qaïda" ou aux talibans $»^{31}$. Une annexe des mesures prises à l'encontre des personnes et groupes de ce dernier cas mentionné contient par exemple la liste des personnes et entités qui sont sous le coup d'un embargo sur le matériel de guerre ou d'une interdiction d'entrée (et de transit) en Suisse. Les institutions qui détiennent ou gèrent des avoirs de ces personnes sont soumises aux sanctions financières; elles doivent donc déclarer les cas suspects au seco. En novembre 2004, 82 comptes bancaires pour un total de 34 millions de francs étaient ainsi bloqués sur la base de l'ordonnance.

\section{SOURCES}

Conseil fédéral, Loi fédérale sur l'Assurance suisse contre les risques à l'exportation (FF 2004 5497).

Conseil fédéral, Message concernant la Loi fédérale sur l'Assurance suisse contre les risques à l'exportation du 24 septembre 2004 (FF 2004 5441).

Conseil fédéral, Rapport du Conseil fédéral sur la politique de maîtrise des armements et de désarmement de la Suisse 2004 (en réponse au postulat Haering 02.3541 du 2 octobre 2002) du 8 septembre 2004 (FF 2004 4833)

Conseil fédéral, Rapport sur la politique économique extérieure 2003 et Message concernant des accords économiques internationaux du 14 janvier 2004 (FF 2004 257).

Conseil fédéral, Rapport sur la politique économique extérieure 2004 et Message concernant des accords économiques internationaux du 12 janvier 2005, disponible sur le site du seco, <www.secoadmin.ch/publikationen/00391/index.html?lang=fr>.

GRE, Rapport annuel 2003, Berne; Zurich, 2004, 46 p.

GRE, Garantie contre les risques à l'exportation. Aperçu, août 2004, 52 p.

Maser B., «De la Garantie contre les risques à l'exportation à l'Assurance suisse contre les risques à l'exportation», La Vie économique, $\mathrm{n}^{\circ} 12,2004$.

seco, Loi fédérale sur l'Assurance suisse contre les risques à l'exportation (LARE). Rapport du DFE sur la procédure de consultation, juin 2004.

economiesuisse, «Une garantie contre les risque à l'exportation moderne pour consolider notre économie», Newsletter, ${ }^{\circ}$ 1-2, janvier-février 2004, p. 3.

Goetschel L., Bernath M. und Schwarz D., Schweizerische Aussenpolitik. Grundlagen und Möglichkeiten, Zürich, Verlag Neue Zürcher Zeitung, 2002. Traduction française: Politique extérieure suisse. Fondements et possibilités, Lausanne, Payot, 2004.

31 Les informations sur les sanctions en cours et sur la politique de la Suisse en la matière figurent sur le site du seco, <www.seco-admin.ch>. Un résumé des changements intervenus dans l'application des sanctions se trouve aussi dans le rapport annuel sur la politique économique extérieure. 
Neue Zürcher Zeitung, «Ausbau des EFTA-Freihandelsnetzes», 29. März 2004; «Die staatliche Exportrisikogarantie im Plus», 23. Juni 2004; «Erweiterter Aktionsradius für die ERG ? », 11. Juli 2004; «Modernisierung der Exportrisikogarantie», 12. Oktober 2004; «Am EFTA-Freihandelsnetz wird weitergeknüpft», 18./19. Dezember 2004.

\section{SITES INTERNET}

Bureau pour la GRE: <www.swiss-erg.com>.

Secrétariat d'Etat à l'économie (Département fédéral de l'économie) : <www.seco-admin.ch>.

ONG : <www.LaDB.ch> et <www.swisscoalition.ch>. 